Artigo Original

\title{
Mudança no foco de atenção ao longo da prática de uma habilidade motora
}

\author{
Cristiane Bhering Moretzsohn Silva ${ }^{2}$ \\ Rodolfo Novellino Benda ${ }^{1}$ \\ Fabiano de Souza Fonseca ${ }^{1}$ \\ João Vitor Alves Pereira Fialho ${ }^{3}$ \\ Hans-Joachim Karl Menzel ${ }^{2}$ \\ Herbert Ugrinowitsch ${ }^{1}$ \\ ${ }^{1}$ Grupo de Estudos em Desenvolvimento e Aprendizagem Motora da Universidade Federal de \\ Minas Gerais, Belo Horizonte, MG,Brasil \\ ${ }^{2}$ Laboratório de Biomecânica, Universidade Federal de Minas Gerais, Belo Horizonte, MG, Brasil \\ ${ }^{3}$ Departamento de Psicologia da Universidade de Warwick, Coventry, Reino Unido
}

Resumo: Este experimento investigou os efeitos da mudança do foco de atenção durante o processo de aprendizagem de uma habilidade motora. Quatro grupos experimentais (foco interno, foco externo, foco interno-externo e foco externo-interno) e um grupo controle praticaram uma tarefa de arremesso de dardo. Durante a fase de aquisição, dois grupos experimentais tiveram uma forma de focalizar a atenção (foco interno ou externo) e os outros dois grupos tiveram o foco de atenção mudado na metade da prática (foco interno-externo ou externo-interno). Os resultados do teste de retenção mostraram que o foco internoexterno resultou em um desempenho mais consistente. Esses resultados sugerem a existência de relação entre os efeitos do foco de atenção e o nível de aprendizagem.

Palavras-chave: Atenção. Habilidade motora. Aprendizagem.

\section{Change in attentional focus through the practice of a motor skill}

Abstract: This experiment investigated the effects of changing the attentional focus during the process of learning a motor skill. Four experimental groups (internal, external, internal-external and external-internal focus) and one control group practiced a dart throwing task. During the acquisition phase, two experimental groups had only one focusing attention (internal or external focus), and other two experimental groups had focus of attention changed half way through the amount of practice (internal-external or external-internal focus). The results of the retention test showed that the internal-external focus resulted in a more consistent performance. These results suggest the existence of a relationship between the effects of attentional focus and level of learning

Keywords: Attention. Motor Skills. Learning.

\section{Introdução}

A informação durante a aprendizagem de habilidades motoras auxilia tanto no fornecimento da ideia do movimento (GENTILE, 1972) quanto para a sua correção (NEWELL, 1974). Como as informações disponíveis no ambiente, juntamente com as fornecidas através das instruções e demonstrações, representam uma sobrecarga para o aprendiz, o direcionamento da sua atenção aos aspectos mais relevantes da tarefa pode ser fundamental para auxiliar a aprendizagem.

No início da prática, o aprendiz recebe instrução e/ou demonstração que englobam informações sobre a posição e coordenação dos membros. Essas informações melhoram o padrão de execução da habilidade (WULF et al., 1998; WULF et al., 1999; WULF; PRINZ, 2001). Uma das explicações é porque as informações direcionadas ao padrão da habilidade induzem a adoção do foco de atenção interno (SHEA; WULF, 1999; WULF et al., 1999; WULF; PRINZ, 2001; TOTSIKA; WULF, 2003; POOLTON et al., 2006; FONSECA; UGRINOWITSCH, 2008). Essa forma de foco de atenção não informa sobre as conseqüências do movimento no ambiente. Por exemplo, em uma habilidade como o arremesso de dardo de salão, as informações com foco interno estariam relacionadas ao movimento do braço ou momento de soltura do dardo.

Quando as informações fornecidas estão relacionadas às consequências do movimento no ambiente, estas induzem a adoção de um foco de atenção externo (SHEA; WULF, 1999; WULF et al., 1999). Nesse caso, as informações podem direcionar a atenção do aprendiz ao implemento, material utilizado ou ao resultado da ação (WULF; 
PRINZ, 2001; WULF, 2007; FONSECA; UGRINOWITSCH, 2008; WULF, 2008). Ao nos reportarmos à mesma tarefa citada anteriormente, as informações com foco externo estariam relacionadas à trajetória do dardo ou à sua posição em relação ao alvo.

Este tópico passou a ser interesse nos estudos de aprendizagem motora a partir do final da década de 1990. Em geral, os resultados evidenciam que o foco externo resulta em melhor desempenho nos testes de retenção e/ou transferência em comparação ao foco interno (WULF et al., 1998; SHEA; WULF, 1999; WULF et al., 2001; MCNEVIN; WULF, 2002; TOTSIKA; WULF, 2003; WULF et al., 2003; WULF et al., 2007; CHIVIACOWSKY et al., 2010; FREUDENHEIM et al., 2010). Esse resultado tem suporte nos resultados de análise eletromiográfica, nos quais o grupo com foco externo apresentou menor atividade eletromiográfica em comparação ao grupo foco interno, sugerindo um menor gasto energético (VANCE et al., 2004; ZACHRY et al., 2005; LOHSE et al., 2010). Uma explicação utilizada para tais resultados é que ao adotar o foco interno, há interferência no controle automatizado do movimento, e consequentemente, ocorre uma degradação da aprendizagem. Já quando é adotado o foco externo, a atenção fica centrada na meta da tarefa, e assim, não há interferência no controle do movimento automatizado e o desempenho é favorecido (SINGER et al., 1993; 1994; WULF; MCNEVIN; SHEA, 2001; MCNEVIN et al., 2003).

Dentre estes estudos, alguns investigaram os efeitos do foco de atenção com diferentes níveis de aprendizagem (iniciantes e habilidosos). Em sujeitos iniciantes, o foco interno propiciou melhor desempenho nos testes de retenção (PERKINSCECCATO et al., 2003; CASTANEDA; GRAY, 2007) e também melhor padrão de execução da tarefa (WULF et al., 2002). Já em sujeitos habilidosos, o foco externo resultou em melhor aprendizagem (WULF et al., 2002; PERKINSCECCATO et al., 2003; WULF; SU, 2007; CASTANEDA; GRAY, 2007). Tais resultados sugerem uma possível relação entre os efeitos do foco de atenção e o nível de aprendizagem. Como na fase inicial de aprendizagem (estágio cognitivo) o desafio do aprendiz é "como fazer a tarefa" (FITTS; POSNER, 1967), o foco interno de atenção pode ser mais eficaz no início da prática. Entretanto, com o decorrer da aprendizagem, gradativamente 0 aprendiz se torna capaz de direcionar sua atenção ao ambiente e elaborar estratégias para alcançar a meta (FITTS; POSNER, 1967). Sendo assim, é possível que o foco externo seja mais eficaz após um tempo de prática da habilidade. Estes resultados indicam que o foco de atenção pode mudar conforme o nível de aprendizagem progride.

Seguindo essa linha de raciocínio, a mudança do foco de atenção durante a prática pode ser uma estratégia capaz de favorecer a aprendizagem. O objetivo do presente estudo foi investigar essa questão e nós testamos a hipótese de que o foco interno no início da prática seguido pelo foco externo resultaria em melhor aprendizagem.

\section{Métodos}

\section{Amostra}

Participaram deste estudo 50 universitários, de ambos os sexos (30 do sexo masculino e 20 do sexo feminino), com idade média de 23,2 $\pm 3,4$ anos, sem experiência na tarefa e todos reportaram ausência de lesão no membro utilizado. Após tomarem conhecimento dos procedimentos experimentais e do objetivo do estudo, todos os voluntários assinaram um termo de consentimento livre e esclarecido.

\section{Tarefa}

Para a coleta de dados foram utilizados dardos oficiais de 20 gramas com ponta metálica e um alvo oficial circular de sisal (Prescott, modelo e medidas oficiais da "BDO - British Darts Organization"), com circunferências circunscritas de $1 \mathrm{~cm}$ de raio e com a pontuação variando de 10 pontos (centro do alvo) a 1 ponto (circunferência mais externa), além de uma televisão 20" e um videocassete. $O$ alvo foi posicionado paralelamente ao solo, com o seu centro distante a 2,5 metros do setor de arremesso. A tarefa consistiu em lançar o dardo com a flexão do ombro no sentido pósteroanterior com o membro superior preferido, abaixo da linha da cintura, com o objetivo de atingir o centro do alvo (Figura 1). Os procedimentos utilizados neste experimento foram aprovados pelo Comitê de Ética Local (ETIC 386/08).

\section{Delineamento e Procedimentos}

Os participantes foram divididos aleatoriamente, mas contrabalançados em relação ao sexo, em cinco grupos: grupo foco interno (Gl), grupo foco externo (GE), grupo combinação foco interno-externo (GIE), grupo 
combinação foco externo-interno (GEI) e grupo controle (GC). O experimento constou de duas fases: aquisição (100 tentativas) e teste de retenção (10 tentativas). A fase de aquisição foi realizada em dois blocos de 50 tentativas, com três minutos de intervalo entre ambos. O teste de retenção foi realizado 10 minutos após o término da fase de aquisição. Antes do início do experimento, foram fornecidas informações gerais sobre a tarefa. Os grupos experimentais receberam demonstrações e instruções que induziam o direcionamento do foco de atenção que serão descritas a seguir. O GC não recebeu nenhum tipo de informação de direcionamento do foco de atenção.

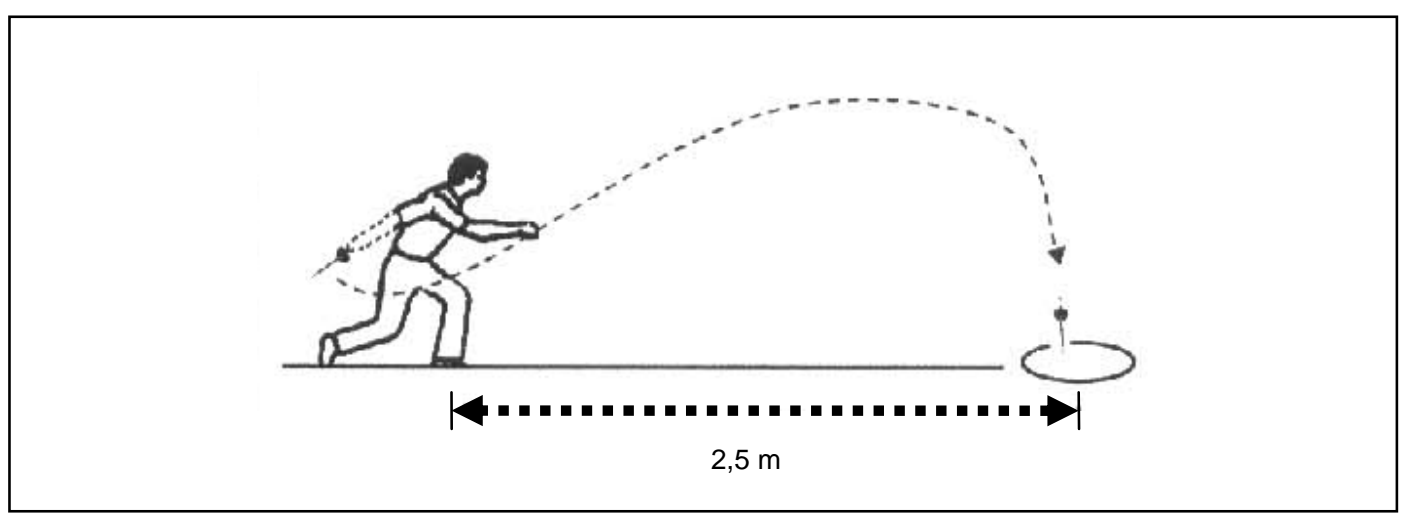

Figura 1. Desenho esquemático da tarefa de arremesso de dardo adaptada de Al-Abood, Davids e Bennett (2001).

As demonstrações foram fornecidas através de vídeo, utilizando um modelo habilidoso que praticou 3.600 vezes a tarefa. Dentre as últimas 300 execuções, as que obtiveram a pontuação 10 foram submetidas a um tratamento de similaridade através da análise hierárquica de Cluster, sendo que as 10 tentativas mais similares (0.9987) foram utilizadas como demonstração. Esse procedimento foi similar ao utilizado por $\underline{\mathrm{Al}-}$ Abood et al. (2001) e Bruzi et al. (2006). As demonstrações com foco interno consistiu na visualização somente do padrão de movimento realizado pelo modelo. Para isso, parte da tela da TV foi coberta para que o voluntário visualizasse exclusivamente 0 movimento realizado pelo modelo. As demonstrações com foco externo consistiu na visualização somente da trajetória do dardo após sair da mão do modelo até atingir o alvo. Parte da tela da TV também foi coberta para que o voluntário não visualizasse o padrão de movimento do modelo. Para garantir que o sujeito mantivesse o foco de atenção desejado, também foram fornecidas instruções antes das demonstrações de acordo com o tipo de foco de atenção desejado (Tabela 1).

Tabela 1. Instruções com dicas de direcionamento do foco de atenção.

\begin{tabular}{ll}
\hline \multicolumn{1}{c}{ FOCO INTERNO } & \multicolumn{1}{c}{ FOCO EXTERNO } \\
\hline 1 - Focalize na perna & 1 - Focalize no dardo \\
2 - Focalize no braço & 2 - Focalize na trajetória do dardo \\
3 - Focalize no cotovelo & 3 - Focalize no alvo \\
\hline
\end{tabular}

As demonstrações e instruções foram fornecidas antes e durante a fase de aquisição. Antes de iniciar a fase de aquisição, os sujeitos receberam nove instruções e dez demonstrações da seguinte forma: a instrução um foi fornecida e, em seguida, foram fornecidas as três primeiras demonstrações; a instrução dois foi fornecida e seguida as demonstrações quatro a seis; e a instrução três foi fornecida e seguida as demonstrações sete a nove. A décima demonstração foi realizada sem instrução. Durante as 50 primeiras tentativas da fase de aquisição, os sujeitos receberam as três instruções e assistiram uma demonstração a cada dez tentativas de prática. Antes de iniciar as últimas 50 tentativas da fase de aquisição, o procedimento inicial foi novamente realizado e os sujeitos receberam mais nove instruções e dez demonstrações. Após isso, a segunda metade da fase de aquisição foi iniciada, adotando os mesmos procedimentos de fornecimento de instrução e demonstração da primeira metade da prática. Esse procedimento garantiu o mesmo número de instruções e demonstrações para os grupos experimentais.

O GE e Gl receberam instruções e demonstrações que induziram somente ao foco de atenção externo e interno, respectivamente. $O$ grupo GEI recebeu instruções e demonstrações com foco externo na primeira metade da fase de aquisição e, na segunda metade, sobre o foco 
interno. O GIE teve o procedimento inverso. O GC não recebeu informação de direcionamento de foco de atenção. No teste de retenção, todos os grupos realizaram a tarefa sem fornecimento de informação que direcionasse o foco de atenção.

A variável dependente utilizada foi o escore obtido a cada arremesso, de acordo com a faixa do alvo na qual $o$ dardo atingia. As faixas variavam em um ponto, que progredia de zero (quando não atingia o alvo) a 10 (quando atingia a faixa central do alvo).

\section{Análise estatística}

As medidas de precisão e de consistência do escore foram analisadas em blocos de cinco tentativas. Para a análise dos dados, duas ANOVA two way com medidas repetidas no segundo fator foram conduzidas, uma na fase de aquisição (5 Grupos x 20 Blocos) e outra no último bloco da aquisição e no teste de retenção (5 grupos $x 5$ blocos). O teste Newman Kleus foi utilizado para identificar possíveis diferenças, e o nível de significância adotado foi $P<0.05$.

\section{Resultados}

\section{a- Precisão nos arremessos}

\section{Fase de Aquisição}

A ANOVA identificou efeito significativo no fator blocos $\left(F_{19,855}=6.398, \quad P<0.001\right)$. $O$ teste de Newman Kleus detectou que o primeiro bloco teve menor pontuação que o segundo $(p=0.049)$ e todos demais blocos $(P<0.001)$. A pontuação do segundo e terceiro blocos foram inferiores à do bloco oito, 10, 12, 14, 15, 16, 18 e $20(P=0.01)$. Não foi identificado efeito significativo no fator grupos $\left(F_{4,45}=1.063, P<0.385\right)$ e nem interação significativa entre grupos e blocos $\left(F_{76,855}=0.863\right.$, $P<0.789$ ) (Figura 2).

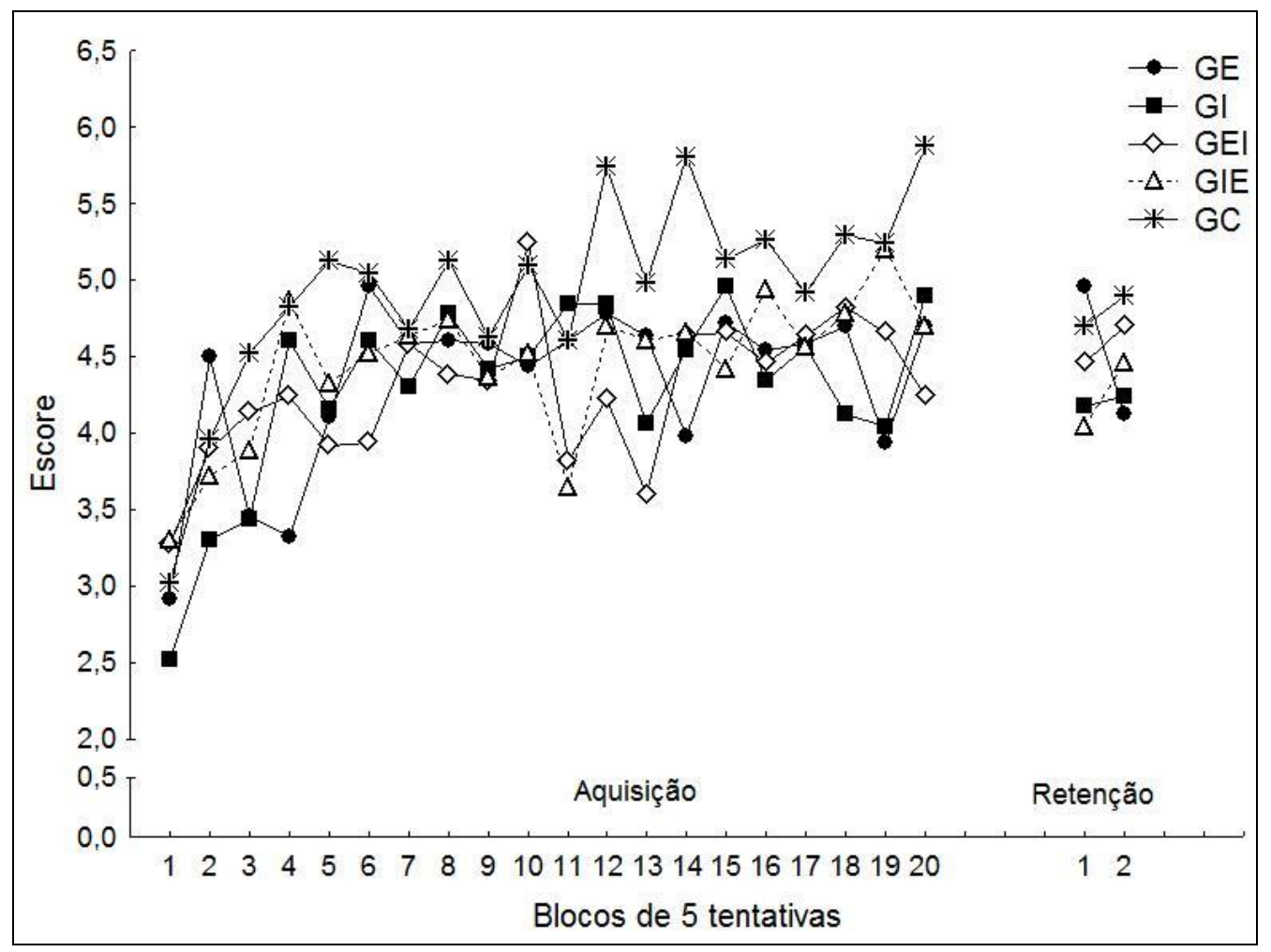

Figura 2. Média do escore dos grupos na fase de aquisição e teste de retenção.

Último bloco da aquisição e teste de retenção

A análise conduzida não identificou efeito significativo no fator grupos $\left(F_{4,45}=0.781, P=\right.$ 0.543) (Figura 2), blocos $\left(F_{2,90}=1.626, P=0.202\right)$ e nem interação significativa entre grupos e blocos $\left(F_{8,90}=0.922, P=0.501\right)$ (Figura 2$)$.

\section{b- Consistência do desempenho}

\section{Fase de Aquisição}

A análise conduzida não identificou efeito significativo no fator grupos, $\left(F_{4,45}=1.704, P<\right.$ $0.165)$, blocos $\left(F_{4,180}=1.445, P<0.097\right)$ e nem interação significativa entre grupos e blocos $\left(F_{16,180}=1.121, P<0.231\right)($ Figura 3$)$. 
Último bloco da aquisição e teste de retenção

A análise não identificou efeito significativo no fator blocos $\left(F_{2,90}=1.207, P=0.917\right)$ e nem interação entre grupos e blocos $\left(F_{8,90}=0.698, P=\right.$
0.692). O teste detectou efeito significativo entre os grupos $\left(F_{4,45}=3.132, P=0.023\right)$, e o teste de Newman Kleus (Figura 3) detectou que o GIE foi mais consistente que o Gl $(P=0.027)$, o GEI $(P=$ $0.046)$ e o $\mathrm{GC}(P=0.030)$ (Figura 3$)$.

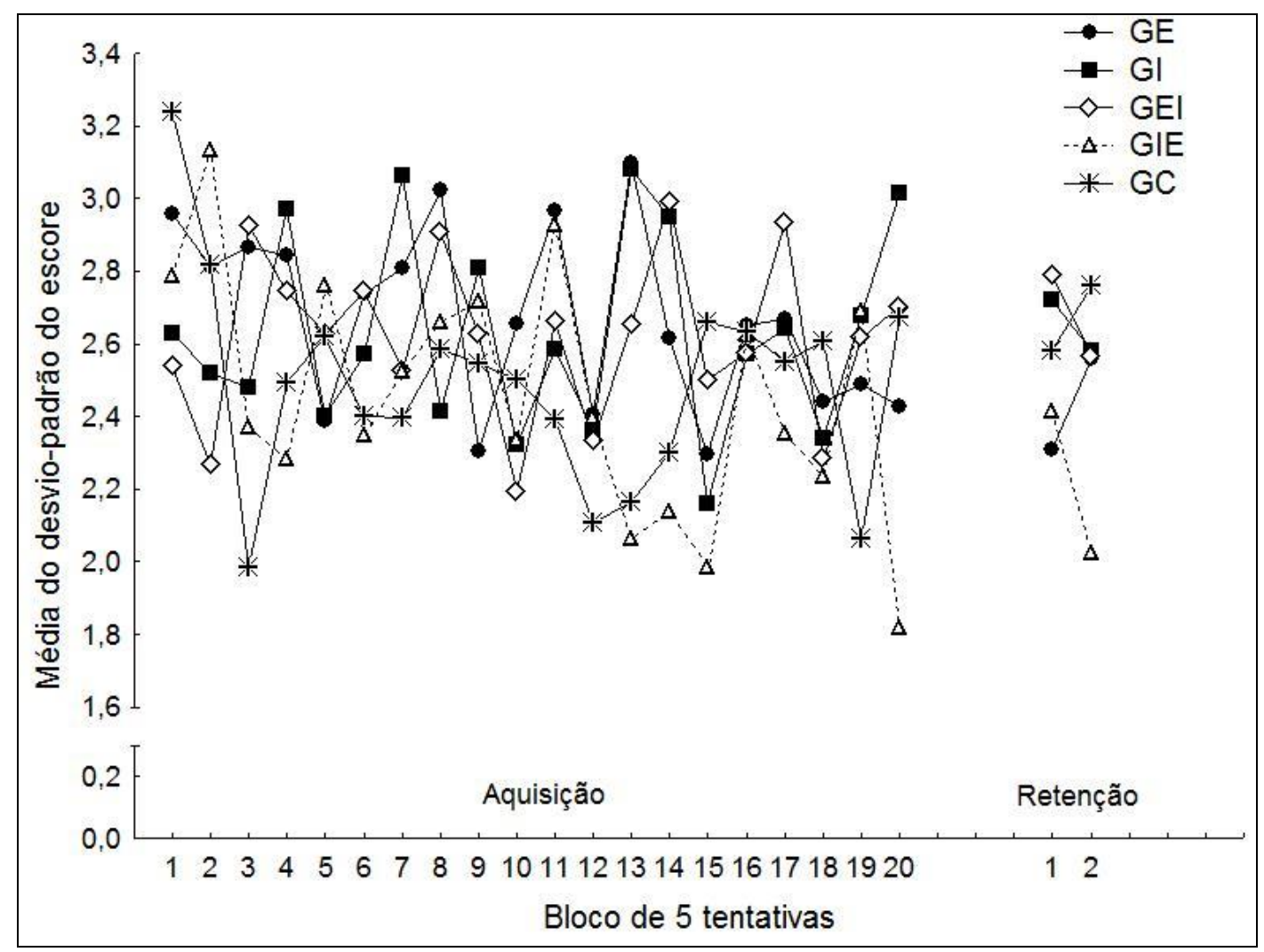

Figura 3. Média do desvio padrão do escore dos grupos na fase de aquisição e teste de retenção.

\section{Discussão}

O objetivo deste estudo foi investigar se a mudança do foco de atenção durante o processo de aprendizagem promoveria efeitos diferentes em função do nível de aprendizagem de uma habilidade motora. A hipótese foi que o foco interno seguido pelo foco externo resultaria em melhor aprendizagem da habilidade praticada. A análise do último bloco da fase de aquisição junto com o teste de retenção mostrou que os grupos mantiveram a precisão no desempenho mesmo após um intervalo sem prática. Esse resultado indica que todos os grupos aprenderam a tarefa, independente da estratégia de foco de atenção utilizada. Entretanto, resultados favoráveis ao GIE foram encontrados na análise da consistência do desempenho.

Tal resultado suporta a hipótese de que a combinação entre informações que induzem ao foco de atenção interno em fases iniciais da aprendizagem, seguidas de informações sobre o foco de atenção externo é uma estratégia capaz de facilitar a aprendizagem. Como em fases iniciais de aprendizagem o aprendiz se envolve mais em aspectos cognitivos da tarefa (FITTS; POSNER, 1967), o foco interno na metade inicial da prática pode ter facilitado esses mecanismos. Por outro lado, em fases mais avançadas o aprendiz gradativamente começa a direcionar a atenção ao meio ambiente (FITTS; POSNER, 1967), e assim, a adoção do foco externo na segunda metade de prática pode ter favorecido nesses processos.

Mesmo não sendo encontrados estudos na literatura que investigaram os efeitos da mudança do foco de atenção durante o processo de aprendizagem, investigações realizadas com indivíduos em diferentes níveis de habilidade suportam os resultados aqui encontrados. Perkins-Ceccato et al., (2003) e Castaneda e Gray (2007) verificaram que a adoção do foco interno para sujeitos iniciantes promoveu melhor aprendizagem em comparação ao foco externo. E ainda, Wulf et al., (2002) também encontraram 
que a adoção do foco interno promoveu melhor aprendizagem do padrão de movimento da habilidade para sujeitos iniciantes. Já para sujeitos habilidosos, a aprendizagem foi facilitada através do foco externo de atenção (WULF et al., 2002; PERKINS-CECCATO et al., 2003; WULF; SU, 2007; CASTANEDA; GRAY, 2007). Sendo assim, nossos resultados avançam nessa questão, demonstrando que a adoção do foco interno no início da prática seguido pelo foco externo é capaz de promover aumento na consistência do desempenho durante a aprendizagem. Com base nos resultados de WULF et al. (2002), PERKINS-CECCATO et al. (2003), WULF; SU (2007), CASTANEDA; GRAY (2007) é possível supor que a maior consistência apresentada pelo GIE esteja relacionada à melhora do padrão da habilidade no início da prática ao adotar o foco interno. Além disso, a melhora na capacidade de parametrização do movimento também parece ter sido induzida pelo foco de atenção externo na segunda metade da prática.

Nossos resultados não evidenciaram superioridade do GIE em relação ao GE, contudo, esse aspecto deve ser discutido. Apesar do GE apresentar desempenho similar ao GIE, ele não conseguiu ser superior aos demais, diferente do GIE. Estes resultados permitem assumir que 0 GIE foi o grupo que teve melhor desempenho do que todos os demais. Esse resultado não dá suporte à hipótese da atenção interrompida (SINGER et al., 1993; 1994; WULF; MCNEVIN; SHEA, 2001; WULF; PRINZ, 2001; MCNEVIN et al., 2003), pois a combinação foco interno-externo parece ter sido importante para maior consistência do desempenho do GIE em relação aos demais. Esse resultado nos leva a assumir a hipótese da relação entre os efeitos do foco de atenção com 0 nível de aprendizagem na habilidade praticada.

Estas diferenças entre os grupos foram encontradas na consistência do desempenho. Esse fato pode ser explicado pelas características da tarefa utilizada no presente estudo. A comparação do arremesso de dardo de salão com as tarefas de balanço no simulador de esqui (WULF et al., 1998), equilíbrio no estabilômetro (WULF et al., 2003) e tacada no golfe (PERKINSCECCATO et al., 2003), indica que ela é uma tarefa relativamente simples do foco de atenção não sejam tão evidentes em tarefas com essas características, pois a demanda de atenção necessária para o controle dos graus de liberdade pode ser baixa. Isso explicaria a ausência de diferença entre o GE, Gl e GC no presente estudo, contrariando as fortes evidências encontradas na literatura de superioridade do foco externo sobre o foco interno e a ausência de direcionamento da atenção (WULF et al., 1998; SHEA; WULF, 1999; WULF et al., 2001; MCNEVIN; WULF, 2002; TOTSIKA; WULF, 2003; CHIVIACOWSKY et al., 2010; WULF et al., 2007; FREUDENHEIM et al., 2010). Esta mesma baixa complexidade da tarefa pode ter favorecido o $\mathrm{Gl}$ e também a ausência de direcionamento da atenção do GC. Indo mais além, por se tratar de uma tarefa relativamente simples, talvez o padrão de movimento no arremesso de dardo não seja tão determinante na precisão do desempenho e a ausência do efeito na medida de precisão do desempenho. por envolver menor número de graus de liberdade. Talvez os efeitos

Os resultados obtidos no presente estudo fornecem indicativos à hipótese de que o foco interno auxilia mais no início da aprendizagem e que o foco externo é melhor após um período de prática. Além disso, também é necessário investigar essa mesma questão em habilidades cujo padrão de movimento tenha uma relação direta com o alcance da meta. A utilização de medidas cinemáticas juntamente com medidas de desempenho pode ser uma importante ferramenta para auxiliar na compreensão desse fenômeno inerente ao processo de aprendizagem motora.

\section{Referências}

AL-ABOOD, S. A.; DAVIDS, K.; BENNETT, S. J. Specificity of task constraints and effects of visual demonstrations in directing learners search during skill acquisition. Journal of Motor Behavior, Washington, v. 33, n. 3, p. 295-305, 2001.

BRUZI, A. T.; PALHARES, L. R.; FIALHO, J. V. A. P.; BENDA, R. N.; UGRINOWITSCH, H. Efeito do número de demonstrações na aquisição de uma habilidade motora: um estudo exploratório.

Revista Portuguesa de Ciências do Desporto, Porto, v. 6, n. 2, p. 179-187, 2006.

CASTANEDA, B.; GRAY, R. Effects of focus of attention on baseball batting performance in players of differing skill level. Journal of Sport \& Exercise Psychology, Champaign, v. 29, n. 1, p. 60-77, 2007.

CHIVIACOWSKY, S.; WULF, G.; WALLY, R. An external focus of attention enhances balance 
learning in older adults. Gait \& Posture, Oxford, v. 32 , n. 4 , p. $572-575,2010$.

FONSECA, F. S.; UGRINOWITSCH, H. Foco de atenção na aprendizagem de habilidades esportivas. In: LEMOS, K. L. M.; SILAMI-GARCIA, E. (ED.) Temas atuais XII. Belo Horizonte: Editora Health, 2008. p. 105-118.

FITTS, P. M.; POSNER, M. I. Human performance. Belmont: Brooks-Cole, 1967.

FREUDENHEIM. A. M.; WULF, G.; MADUREIRA, F.; PASETTO, S. C.; CORRÊA, U. C. An external focus of attention results in greater swimming speed. International Journal of Sports Science and Coaching, Leeds, v. 5, n. 4, p. 533-542, 2010.

GENTILE, A. M. A working model of skill acquisition with application to teaching. Quest, Champaign, v. 17, n. 1, p. 3-23, 1972.

LOHSE, K. R.; SHERWOOD, D. E.; HEALY, A. F. How changing the focus of attention affects performance, kinematics, and electromyography in dart throwing. Human Movement Science, Amsterdan, v. 29, n. 4, p. 542-555, 2010.

McNEVIN, N. H.; WULF, G. Attentional focus on supra-postural tasks affects postural control. Human Movement Science, Amsterdan, v. 21, n. 2, p. 187-202, 2002.

MCNEVIN, N. H.; SHEA, C. H.; WULF, G. Increasing the distance of an external focus of attention enhances learning. Psychological

Research, Berlin, v. 67, n. 1, p. 22-29, 2003.

NEWELL, K. M. Knowledge of results and motor learning. Journal of Motor Behavior, Washington, v. 6, n. 4, p. 235-244, 1974.

PERKINS-CECCATO, N.; PASSMORE, S. R.; LEET, D. Effects of focus of attention depend on golfers' skill. Journal of Sports Sciences, London, v. 21, n. 8, p. 593-600, 2003.

POOLTON, J. M.; MAXWELL, J. P.; MASTERS, R. S.; RAAB, M. Benefits of an external focus of attention: common coding or conscious processing? Journal of Sports Sciences, London, v. 24, n. 1, p. 89-99, 2006.

SHEA, C. H.; WULF, G. Enhancing motor learning through external focus instructions and feedback. Human Movement Science, Amsterdan, v. 18, n. 4, p. 553-571, 1999.

SINGER, R. N.; LIDOR, R.; CAURAUGH, J. H. To be aware or not aware: what to think about while learning and performing a motor skill. The Sport Psychologist, Sheffield, v. 7, n. 1, p. 19-30, 1993.
SINGER, R. N.; LIDOR, R.; CAURAUGH, J. H. Focus of attention during motor skill performance. Journal of Sports Sciences, London, v. 12, n. 4, p. 335-340, 1994.

TOTSIKA, V.; WULF, G. An external focus of attention enhances transfer to novel situations and skills. Research Quarterly for Exercise and Sport, Washington, v. 74, n. 3, p. 220-225, 2003.

VANCE, J.; WULF, G.; TÖLLNER, T.; McNEVIN, N.; MERCER J. EMG activity as a function of the performer's focus of attention. Journal of Motor Behavior, Washington, v. 36, n. 4, p. 450-459, 2004.

WULF, G. Attention and Motor Skill Learning. Champaign: Human Kinetics, 2007.

WULF, G. Attentional focus effects in balance acrobats. Research Quarterly for Exercise and Sport, Washington, v. 79, n. 3, p. 319-325, 2008.

WULF, G.; PRINZ, W. Directing attention to movement effects enhances learning: A review. Psychonomic Bulletin \& Review, Austin, v. 8, n. 4, p. 648-660, 2001.

WULF, G.; SU, J. External focus enhances golf shot in beginners and experts. Research Quarterly for Exercise and Sport, Washington, v. 78, n. 4, p. 384-389, 2007.

WULF, G.; HOB, M.; PRINZ, W. Instructions for motor learning: differential effects of internal versus external focus of attention. Journal of Motor Behavior, Washington, v. 30, n. 2, p. 169179, 1998.

WULF, G.; LAUTERBACH, B.; TOOLE, T. Learning advantages of an external focus of attention in golf. Research Quarterly for Exercise and Sport, Washington, v. 70, n. 2, p. 120-126, 1999.

WULF, G.; McNEVIN, N. H.; SHEA, C. H. The automaticity of complex motor skill learning as a function of attentional focus. Quarterly Journal of Experimental Psychology, London, v. 54, n. 4, p. 1143-1154, 2001.

WULF, G.; TOLLNER, T.; SHEA, C. H. Attentional focus effects as a function of task difficulty Research Quarterly for Exercise and Sport, Washington, v. 78, n. 3, p. 257-264, 2007.

WULF, G.; McCONNEL, N.; GARTNER, M.; SCHWARZ, A. Enhancing the learning of sport skills through external-focus feedback. Journal of Motor Behavior, Washington, v. 34, n. 2, p. 171182, 2002. 
WULF, G.; WEIGELT, M.; POULTER,

D.; McNEVIN, N. Attentional focus on

suprapostural tasks affects balance learning.

Quarterly Journal of Experimental Psychology,

London, v. 56, n. 6, p. 1191-1211, dec. 2003.

ZACHRY, T; WULF, G.; MERCER, J.; BEZODIS,

$\mathrm{N}$. Increased movement accuracy and reduced

EMG activity as the result of adopting an external

focus of attention. Brain Research Bulletin,

Phoenix, v. 67, n. 4, p. 304-309, 2005.

Agências de Financiamento: $O$ projeto foi realizado com suporte do Programa Pesquisador Mineiro IV (PPM-00084-10).

\section{Endereço:}

Fabiano de Souza Fonseca

R. Vitório Magnavacca, 251/301-Bloco 3 Buritis

Belo Horizonte MG Brasil

30455-730

Telefone: (31) 94694037

e-mail: fabianoef@gmail.com

Recebido em: 21 de abril de 2012.

Aceito em: 19 de abril de 2013.

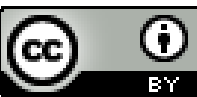

Motriz. Revista de Educação Física. UNESP, Rio Claro, SP, Brasil - elSSN: 1980-6574 - está licenciada sob Creative Commons - Atribuicãa 3.0 